\title{
Identifying improvements in an emergency department elder friendly area based on patient experience "ED_EFA their voice project"
}

\author{
Mireia Puig ${ }^{1,3,4}$, Miriam Mateo ${ }^{1,3,4}$, Marta Blázquez ${ }^{1,3,4}$ and Josep Ris ${ }^{1,2,4}$ \\ ${ }^{1}$ Emergency Department, Hospital de la Santa Creu i Sant Pau, Barcelona, Spain \\ ${ }^{2}$ Urgent Care Process, Hospital de la Santa Creu i Sant Pau, Barcelona, Spain \\ ${ }^{3}$ Universitat Autònoma de Barcelona, Medicine Department. Barcelona, Spain \\ ${ }^{4}$ IIB Sant Pau Research Institute, Barcelona, Spain
}

\section{Introduction}

The Emergency Departments (EDs) classically focus on the identification of patients' acute disorders, their diagnosis, treatment and early discharge. However, in older people, urgent care must have particular features to ensure quality [1]. First, it frequently requires simultaneous treatment of several medical, social and functional problems. In addition, the priorities are symptom control, maximizing quality of life, and ensuring the continuum of care. For all these reasons, experts and scientific societies recommend developing strategies in EDs that combine: 1) comprehensive geriatric assessment (CGA); 2) structural customization; and 3) training of professionals in geriatric care [2-5].

In this regard, Hospital de la Santa Creu i Sant Pau ED has developed a Program of Care for frailty (PCF) over the last 10 years, which includes nursing care and proceedings focused on older people, and a territorial network with other healthcare providers [6]. In November 2017, the program's implementation process was completed with the creation of an Elderly Friendly Area (ED_EFA). All this has led us to obtain in May 2019, the first GEDA (Geriatric Emergency Department Accreditation) that the ACEP (American College on Emergency Physicians) has delivered to an ED outside the United States or Canada [7].

One of PCF's major objectives is the prevention and proper management of delirium, which is a geriatric syndrome that frequently appears in elderly patients in the ED and has been associated with a poor prognosis (increased stays, higher admission rate and mortality) [8]. In this way, the nursing care plan and the structural elements of the area can bring benefits. For this reason, the new EFA was built, with adjustable natural and artificial light, soundproofing, light-absorbing colours that prevent reflections that may precipitate hallucinations in patients with age-related visual defects, adequate furniture that maximizes the comfort, comfortable armchairs, furniture and adequate space for the companion, circadian rhythms in the procedures that allow night's rest, individual boxes with an autonomous buzzer to warn the unit's professionals. It is therefore an observation area integrated into the Emergency Department where the old people wait between 12-24 hours until the diagnostic process has been completed or there has been clinical improvement and they can be transferred to the best destination for discharge (home, hospitalization at home, nursing home, or socio-sanitary centers) $[6,8]$.

Our Program of Care for Frailty has proved its worth in organizational and operational terms [6]. However, we aimed to carefully evaluate patients' experience in the new EFA. According to the Beryl Institute's definition, the patient experience is the sum of all interactions, shaped by an organization's culture, that influence patient perceptions throughout the continuum of care [9].

This project aims to analyse the quality of patients experiences in ED_EFA and identify areas for improvement and feasible solutions. The project uses the creative problem-solving methodology called Design Thinking.

Design Thinking is a creativity and innovation methodology that compiles tools for problem solving based on design principles. It is characterized by its ability to mix convergent and divergent thinking, reconciling rational and intuitive thinking. This methodology consists of four phases: Mapping (process mapping), Exploration (revision of the process), Construction (creation of new proposals) and Testing (implementation of these). In every step, techniques help to observe, get involved and immerse ourselves in the ecosystem of the patient, with the aim of designing a solution with the user and not just for him $[10,11]$.

During the project, an assessment questionnaire was designed and distributed among ED professionals, EFA users and their caregivers. A high level of satisfaction was observed. Despite this, it was found that there were opportunities for improvement in patient information about their process and also about the peculiarities of the area. In a very special way, it was proposed to facilitate a greater involvement of the families in patient care. A co-creation workshop was held with professionals, patients and families, which resulted in the production of two prototypes. The first one refers to a leaflet or Engagement Card, which provides information to patients and relatives about the EFA peculiarities. In addition, it includes a set of proposals through which the relative may volunteer to take part in the care (meals, entertainment and hygiene). The second prototype is a customized board containing

${ }^{\star}$ Correspondence to: Mireia Puig Campmany, Emergency Department, Hospital de la Santa Creu I Sant Pau, Spain, E-mail: mpuigc@santpau.cat

Key words: emergency department, elder, frailty

Received: May 29, 2019; Accepted: June 06, 2019; Published: June 10, 2019 
details of ED team and clinical process. At this moment the project is in a revision phase after the implantation of the two prototypes, and we hope to observe an improved experience of our users.

In summary, EDs must adapt to care for the older and more vulnerable population, which is subject to increasing risks during its stay and suffers particularly negative experiences in this setting. Adaptation requires the integration of comprehensive geriatric assessment and multidisciplinary care provided by emergency professionals with specific skills. It also requires guaranteeing quality transitions. The main objective of the ED organization is to achieve an efficient, safe and high-quality care process. In this sense, the patient's experience is an added dimension. The organization's knowledge of this aspect and including the patient's point of view can create more ergonomic processes, tailored to the patients' needs and lead to higher quality experiences.

\section{Acknowledgements}

The authors acknowledge the nurses, support staff, physicians, social workers, pharmacist and leadership at the Hospital de la Santa Creu i Sant Pau Emergency Department and other health providers in AIS Dreta; their enthusiastic efforts enabled the creation of a frailty program for patients to receive better urgent care.

Research group: Boukdra Attou, Marta Blázquez, Marco Bustamante, Hector Hernández, Sergio Herrera, J.Leopoldo Higa, Yaiza Ibáñez, Miriam Mateo, $M^{a}$ Dolores Muñoz, Silvia Nuño, Carla Pedragosa, Mar Pina, Mireia Puig, Josep Ris, Carme Valls, Miquel Angel Viciana.

\section{Compliance with ethical standards}

\section{Conflict of interest}

The authors state that there is no conflict of interest and that no commercial, financial, and other relationships exist. This project received the Premio Quirónsalud a las Mejores Iniciativas en Experiencia del Paciente - Primera Edición, 2018 (QuironSalud Award for Best Patient Experience Initiatives, First Edition, 2018).

\section{Ethical approval}

In this article, patient data do not appear. The clinical research ethics committee of reference (Ethics Committee Sant Pau Biomedical
Research Institute, IIB Sant Pau, Barcelona) approved this study (IIBSPEXP-2018-80). For this type of study informed consent is not required. ClinicalTrials.gov Identifier: NCT03717324.

\section{Proponent}

The project is developed in collaboration with Merck Sharp \& Dohme (MSD) of Spain, under an agreement card (Fundació de Gestió Hospital de la Santa Creu i Sant Pau - MSD). MSD has hired a specialist company to develop this kind of dynamics (Thinkers\&Co). No monetary payment is made to the hospital, to the emergency department or to the professionals involved.

\section{References}

1. Rosenberg MS, Carpenter CR, Bromley M, Caterino JM, Chun A, et al. (2014) Geriatric emergency department guidelines. Ann Emerg Med 63: e7-e25. [Crossref]

2. Banerjee J, Conroy S, Cooke MW (2013) Quality care for older people with urgent and emergency care needs in UK emergency departments. Emerg Med J 30: 699-700. [Crossref]

3. Burton JH, Young J, Bernier CA (2014) The Geriatric ED: Structure, Patient Care, and Considerations for the Emergency Department Geriatric Unit. International Journal of Gerontology 8: 56e-59.

4. Keyes DC, Singal B, Kropf CW, Fisk A (2014) Impact of a new senior emergency department on emergency department recidivism, rate of hospital admission, and hospital length of stay. Ann Emerg Med 63: 517-524. [Crossref]

5. Conroy S, Nickel CH, Jónsdóttir AB, Fernandez M, Banerjee J, et al. (2016) The development of a European curriculum in Geriatric Emergency Medicine. Eur Geriat Med 7: 315-321

6. Puig M, Ris J, Blázquez M, Benito S (2019) Developement of a comprehensive, multidisciplinary program of care for frailty in an Emergency Department. Eur Geriatr Med 10: 37-46.

7. Geriatric Emergency Department Accreditations. ACEP Geriatric. Available at: https:// www.acep.org/geda/

8. Bo M, Bonetto M, Bottignole G, Porrino P, Coppo E, et al. (2016) Length of Stay in the Emergency Department and Occurrence of Delirium in Older Medical Patients. $\mathrm{Am}$ Geriat Soc 64: 1114-1119. [Crossref]

9. The Beryl Institute. The patient experience definition. Available at: https://www. theberylinstitute.org/page/CopyofDefiningPati

10. Stopping Hospital Infections in Their Tracks. Taking a personalized hand-sanitizing device to the next level. 2013. Available at: https://www.ideo.com/case-study/ stopping-hospital-infections-in-their-tracks

11. Kim SH, Myers CG, Allen L (2017) Health Care Providers Can Use Design Thinking to Improve Patient Experiences. Available at: https://hbr.org/2017/08/health-careproviders-can-use-design-thinking-to-improve-patient-experiences

Copyright: (C2019 Puig M. This is an open-access article distributed under the terms of the Creative Commons Attribution License, which permits unrestricted use, distribution, and reproduction in any medium, provided the original author and source are credited. 